\title{
己内酰胺功能化离子液体的合成及其催化酯化性能的研究
}

\author{
胡晶晶赵地顺* 李静静翟建华胡甜甜 \\ (河北科技大学化学与制药工程学院 石家庄 050018)
}

\begin{abstract}
摘要 以价格低廉的己内酰胺为原料, 制备和表征了 4 种己内酰胺功能化离子液体: 1-(3-磺丙基)己内酰胺硫酸氢盐 $\left(\left[\mathrm{C}_{3} \mathrm{SO}_{3} \mathrm{HCP}\right] \mathrm{HSO}_{4}\right) 、 1-\left(3\right.$ - 磺丙基) 己内酰胺对甲苯磺酸盐 $\left(\left[\mathrm{C}_{3} \mathrm{SO}_{3} \mathrm{HCP}\right] \mathrm{PTSA}\right) 、 1-(3$-磺丙基) 己内酰胺磷酸氢盐 $\left(\left[\mathrm{C}_{3} \mathrm{SO}_{3} \mathrm{HCP}\right] \mathrm{H}_{2} \mathrm{PO}_{4}\right) 、 1-(3$-磺丙基 $)$ 己内酰胺四氟硼酸盐 $\left(\left[\mathrm{C}_{3} \mathrm{SO}_{3} \mathrm{HCP}\right] \mathrm{BF}_{4}\right)$. 以乙酸和乙醇的酯化反应考察 4 种酸性离子 液体的催化活性, 并与 3 种具有不同氮杂环的 $\mathrm{SO}_{3} \mathrm{H}$-功能化离子液体和浓硫酸相对照. 结果表明: 当 $n\left(\mathrm{C}_{2} \mathrm{H}_{5} \mathrm{OH}\right)$ : $n\left(\mathrm{CH}_{3} \mathrm{COOH}\right)=1: 1.5$, 催化剂 $\left[\mathrm{C}_{3} \mathrm{SO}_{3} \mathrm{HCP}\right] \mathrm{HSO}_{4}$ 用量为酸醇总质量的 $5 \%$, 反应温度 $80{ }^{\circ} \mathrm{C}$, 反应时间 $6 \mathrm{~h}$, 酯收率可达 93.8\%, 离子液体经真空干燥重复使用 10 次后, 仍具有较高的催化活性, 而且对奥氏体 $316 \mathrm{~L}$ 不锈钢试样的腐蚀率不到 浓硫酸的 $1 / 6$. 还考察了以 $\left[\mathrm{C}_{3} \mathrm{SO}_{3} \mathrm{HCP}\right] \mathrm{HSO}_{4}$ 为催化剂催化合成系列乙酯也获得了较高的酯收率, 且离子液体均能与酯 产物自动分相. 与传统硫酸催化酯化相比，此类离子液体催化酯化具有生产成本低、过程清洁、腐蚀率低、使用周期 长等优点, 具有替代传统浓硫酸催化醇酸酯化反应的潜力.
\end{abstract}

关键词 己内酰胺; 功能化离子液体; 酯化反应; 催化活性

\section{Synthesis and Catalytic Esterification Performance of Caprolactam Task-Specific Ionic Liquids}

\author{
$\mathrm{Hu}$, Jingjing \\ Zhao, Dishun* \\ Li, Jingjing \\ Zhai, Jianhua \\ Hu,Tiantian \\ (College of Chemical and Pharmaceutical Engineering, Hebei University of Science and Technology, Shijiazhuang 050018)
}

\begin{abstract}
Four kinds of caprolactam task-specific ionic liquids (TSILs), 1-(3-sulfopropyl)caprolactam hydrogen sulfate, 1-(3-sulfopropyl)caprolactam $p$-toluene sulfonate, 1-(3-sulfopropyl)caprolactam phosphate sulfonate, 1-(3-sulfopropyl)caprolactam tetrafluoroborate, were prepared and characterized with low price caprolactam as raw material. The catalytic activities of these acidic ionic liquids were investigated by the esterification of acetic acid with ethanol and were compared with 3 kinds of $\mathrm{SO}_{3} \mathrm{H}$-functionalized ionic liquids with different nitrogen heterocycles and concentrated sulfuric acid. The results showed that under the optimized conditions $\left[n\left(\mathrm{C}_{2} \mathrm{H}_{5} \mathrm{OH}\right): n\left(\mathrm{CH}_{3} \mathrm{COOH}\right)=1: 1.5\right]$, catalyst amount of $5 \%(\mathrm{w})$ based on acetic acid and ethanol., reaction temperature $80{ }^{\circ} \mathrm{C}$ and reaction time $6 \mathrm{~h}$ ), the yield of ethyl acetate was up to $93.8 \%$. The ionic liquids dried in vacuum till remained high activity after reused for 10 times, and the corrosion rates of the $316 \mathrm{~L}$ austenitic stainless steel plates immersed in the systems with these ionic liquids were less than one sixth of that with sulfuric acid. Finally, the yields of series of ethyl esters were also high with $\left[\mathrm{C}_{3} \mathrm{SO}_{3} \mathrm{HCP}\right] \mathrm{HSO}_{4}$ as catalyst, and the ionic liquids can form split-phases with ester product. Compared with the traditional sulfuric acid catalytic esterification, catalytic esterification of such ionic liquid has the advantages of low production cost, clean process, low corrosion rate, long useage cycle, and has the potential of replacing the traditional sulfuric acid in catalytic esterification reaction.
\end{abstract}

Keywords caprolactam; task-specific ionic liquids; esterification; catalytic activity

有机羧酸酯是重要的精细化工产品, 常用作溶剂和 香料，可用于合成香料、化妆品、食品及饲料添加剂、 表面活性剂、防腐防霉剂、橡胶及塑料的增塑剂、制药 工业中的原料和中间体等，在国内外具有广阔的需求市
场. 合成有机羧酸酯的传统工艺是采用浓硫酸催化醇酸 酯化，该法引起的副反应多、产物收集过程复杂，其工 业化的催化剂浓硫酸对设备严重腐蚀，易造成三废污 染，因此寻找绿色、廉价的催化剂来代替浓硫酸尤为重

\footnotetext{
* E-mail: zhao_dsh@hebust.edu.cn; Tel.: 0311-88632231; Fax: 0311-88632231

Received March 10, 2015; revised April 21, 2015; published online April 27, 2015.

Project supported by the National Natural Science Foundation of China (Nos. 20576026, 21106032).

国家自然科学基金(Nos. 20576026, 21106032)资助项目.
} 
要 $^{[1,2]}$. 近年来, 研究者们在寻找可代替硫酸的新型酯化 催化剂方面做了大量工作, 开发出一系列优良的醇酸酯 化反应催化剂 ${ }^{[3 \sim 6]}$.

随着新型离子液体的不断涌现, 离子液体从 “耐水 体系” 向 “功能体系” 发展, 研究发现功能化离子液体 同时具备固体酸和液体酸的优点, 因此更多的研究已集 中于这种环境友好体系在催化、合成、萃取-分离、电 化学等领域的应用开发上 ${ }^{[7 ~ 15]}$. 将咪唑类离子液体用作 新兴的反应介质已经成为研究热点, 它可以作为催化 剂/溶剂用于各类酯化反应中, 得到了较好的催化效果, 但离子液体用量过多, 且咪唑基离子液体的合成需要使 用价格昂贵的甲基咪唑作为基本原料, 而且咪唑基离子 液体具有一定的毒性 ${ }^{[16]}$, 因此合成廉价、环境友好的离 子液体是室温离子液体研究的一个重要方向. 在离子液 体的合成方面除了对普通的咪唑基、吡啶基室温离子液 体进行修饰外, 还出现了几类新型的可作为离子液体的 阳离子和阴离子. 其中质子化内酰胺阳离子就是一种新 型阳离子, Deng 等 ${ }^{[17]}$ 以价格低廉的内酰胺(己内酰胺、 丁内酰胺) 作为原料, 合成了一系列质子化内酰胺 Brønsted 酸性室温离子液体, 初步实验结果表明该类 内酰胺基 Brønsted 酸性离子液体在室温下呈液态或熔 点小于 $70{ }^{\circ} \mathrm{C}$, 有较强的酸性, 能作为酸性催化剂或反 应介质用于许多传统的酸催化反应中. 目前, 质子化内 酰胺类 Brønsted 酸性离子液体催化酯化反应的研究还 鲜有报道，且制备己内酰胺类 Brønsted 酸性离子液体 催化剂所需的原料成本低, 市场供应量大, 有一定的应 用前景. 本文以价格低、毒性小的己内酰胺为原料合成 了 4 种新型的己内酰胺 Brønsted 酸性离子液体(Scheme 1), 考察了其催化合成乙酸乙酯的催化活性, 并对反应 条件进行了优化. 同时考察了 $\left[\mathrm{C}_{3} \mathrm{SO}_{3} \mathrm{HCP}\right] \mathrm{HSO}_{4}$ 对系列 乙酯的合成, 表现出较强的催化性能, 克服了传统催化 剂存在的缺点, 提高了生产效率, 降低了生产成本, 有 望代替传统酸性催化剂，具有广阔的应用前景.

\section{1 结果与讨论}

\section{1 离子液体的酸性分析}

酸性离子液体不仅具有液体酸的高密度反应活性 位, 又有固体酸的不挥发性. 酯化反应作为酸催化的可 逆反应, 其反应进行的程度与催化剂的酸性强弱有直接 的关系, 而离子液体的催化活性是由离子液体提供的酸 性来决定的, 酸性越强, 催化活性越好. 表 1 为不同浓 度(质量分数)的离子液体与浓硫酸的水溶液的 $\mathrm{pH}$ 值.

由表 1 可以看出, 对于同一种溶液, 其 $\mathrm{pH}$ 值随着质 量浓度的增大而降低, 因为水有高的介电常数以及很强 的与离子液体阴离子形成氢键的能力, 从而能有效地解<smiles>CCC1=[N+](CCCS(=O)(=O)[O-])CCCCC1=O</smiles>

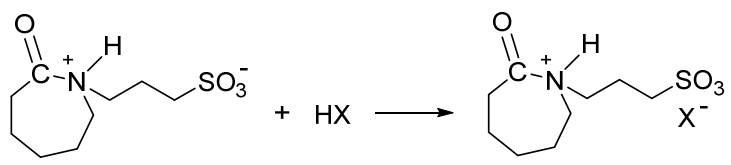<smiles></smiles>

$\left[\mathrm{C}_{3} \mathrm{SO}_{3} \mathrm{HCP}\right] \mathrm{HSO}_{4}$

IL1<smiles>C[N+]1(CCCS(=O)(=O)O)CCCCCC1=O</smiles>

$\left[\mathrm{C}_{3} \mathrm{SO}_{3} \mathrm{HCP}\right] \mathrm{H}_{2} \mathrm{SO}_{4}$

IL3<smiles>CPCCCC1CCCCC(=O)N1</smiles>

$\left[\mathrm{C}_{3} \mathrm{SO}_{3} \mathrm{HCP}\right] \mathrm{PTSA}$

IL2<smiles>O=C1CCCCCC1NCCCS(=O)(=O)O</smiles>

$\left[\mathrm{C}_{3} \mathrm{SO}_{3} \mathrm{HCP}\right] \mathrm{PTSA}$

IL4
图式 1 离子液体的合成

Scheme 1 Synthesis of ionic liquids

表 1 不同离子液体的酸性 ${ }^{a}$

Table 1 The acidity of various ionic liquids in water

\begin{tabular}{lccccc}
\hline \multirow{2}{*}{\multicolumn{1}{c}{ Compd. }} & \multicolumn{5}{c}{$\mathrm{pH}$} \\
\cline { 2 - 6 } & $1 \%^{b}$ & $2 \%^{b}$ & $3 \%^{b}$ & $4 \%^{b}$ & $5 \%^{b}$ \\
\hline$\left[\mathrm{C}_{3} \mathrm{SO}_{3} \mathrm{Hmim}\right] \mathrm{HSO}_{4}$ & 1.32 & 1.23 & 1.06 & 0.82 & 0.65 \\
{$\left[\mathrm{C}_{3} \mathrm{SO}_{3} \mathrm{HPy}\right] \mathrm{HSO}_{4}$} & 1.35 & 1.24 & 1.07 & 0.84 & 0.67 \\
{$\left[\mathrm{C}_{3} \mathrm{SO}_{3} \mathrm{Hnhm}\right] \mathrm{HSO}_{4}$} & 1.34 & 1.24 & 1.05 & 0.85 & 0.66 \\
{$\left[\mathrm{C}_{3} \mathrm{SO}_{3} \mathrm{HCP}\right] \mathrm{HSO}_{4}$} & 1.33 & 1.23 & 1.04 & 0.83 & 0.65 \\
{$\left[\mathrm{C}_{3} \mathrm{SO}_{3} \mathrm{HCP}\right] \mathrm{PTSA}$} & 1.49 & 1.34 & 1.25 & 1.07 & 0.73 \\
{$\left[\mathrm{C}_{3} \mathrm{SO}_{3} \mathrm{HCP}\right] \mathrm{H}_{2} \mathrm{PO}_{4}$} & 1.57 & 1.41 & 1.30 & 1.12 & 0.88 \\
{$\left[\mathrm{C}_{3} \mathrm{SO}_{3} \mathrm{HCP}\right] \mathrm{BF}_{4}$} & 1.65 & 1.54 & 1.42 & 1.26 & 0.93 \\
$\mathrm{H}_{2} \mathrm{SO}_{4}$ & 1.32 & 1.25 & 1.03 & 0.81 & 0.64 \\
\hline
\end{tabular}

${ }^{a}$ Test condition: $T=15{ }^{\circ} \mathrm{C} .{ }^{b} w(\mathrm{IL}) / \%$.

离离子液体的聚集体; 阴离子相同的离子液体, $\mathrm{SO}_{3} \mathrm{H}-$ 功能化离子液体和浓硫酸的水溶液的 $\mathrm{pH}$ 值都很相近, 说明离子液体酸性强弱与氮杂环结构没有呈现明显的 相关性; 阳离子相同的离子液体，其 $\mathrm{pH}$ 值随阴离子的 酸性的增强而增大，虽然丙磺酸基的引入增加了一个酸 性位，使酸性增强，但差别不是很大，说明阴离子对离 子液体的酸性的影响作用更大. 通过对离子液体酸强度 的测试, 为后续对催化剂催化性能的研究提供了理论依 据.

\section{2 离子液体的结构分析}

4 种已内酰胺功能化离子液体的红外谱图数据如图 2, 由图 2 可知, $3280 \sim 2940 \mathrm{~cm}^{-1}$ 内出现的为 $\mathrm{N}-\mathrm{H}$ 的伸 缩振动峰, $1600 \mathrm{~cm}^{-1}$ 左右出现的为 $\mathrm{N}-\mathrm{H}$ 的弯曲振动 


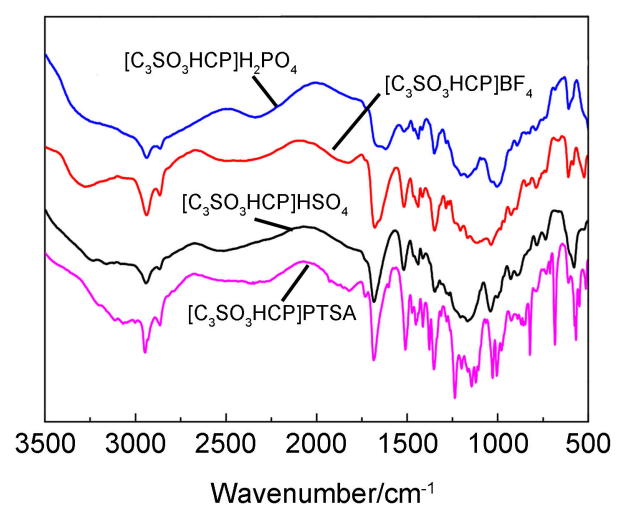

图 2 离子液体的红外谱图

Figure 2 The IR spectra of ionic liquids

峰; $2950 \sim 2850 \mathrm{~cm}^{-1}$ 内出现的为 $\mathrm{C}-\mathrm{H}$ 伸缩振动峰, 1485 1445 $\mathrm{cm}^{-1}$ 内出现的为 $\mathrm{C}-\mathrm{H}$ 面内弯曲振动峰; $1700 \sim 1600 \mathrm{~cm}^{-1}$ 内出现的为 $\mathrm{C}=\mathrm{O}$ 伸缩振动峰; $870 \mathrm{~cm}$ ${ }^{-1}$ 左右出现的为 $\mathrm{S}=\mathrm{O}$ 伸缩振动峰, $528 \mathrm{~cm}^{-1}$ 左右出现 的为 $\mathrm{S}=\mathrm{O}$ 弯曲振动峰; $3429 \mathrm{~cm}^{-1}$ 左右出现的为 $\mathrm{O}-\mathrm{H}$ 伸缩振动峰, $1236 \mathrm{~cm}^{-1}$ 左右出现的为 $\mathrm{O}-\mathrm{H}$ 键弯曲振动 峰; $\mathrm{HSO}_{4}^{-} 、 \mathrm{SO}_{3} \mathrm{H} 、 \mathrm{H}_{2} \mathrm{PO}_{4}^{-}$和 $\mathrm{BF}_{4}^{-}$的特征吸收峰分别 出现在 1250、1160 1040、489、1036 cm $\mathrm{cm}^{-1}$ 处. 综上可
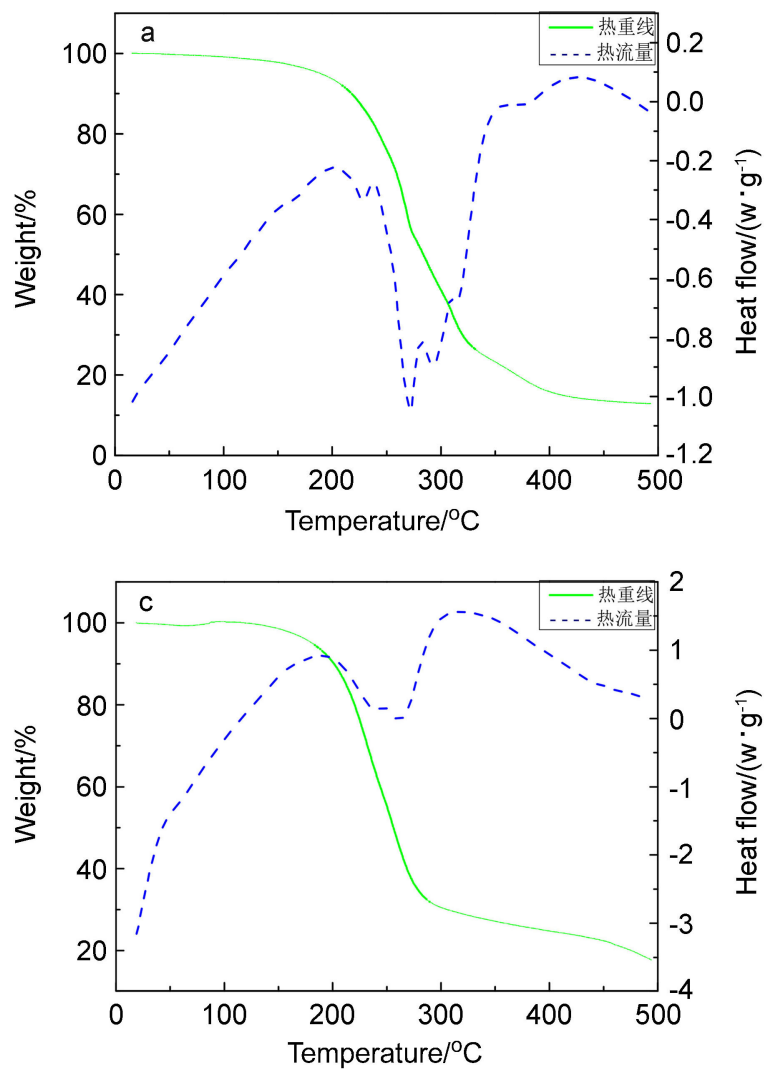

知, 4 种新型己内酰胺类离子液体的结构得到了初步验 证.

\section{3 离子液体的热稳定性}

离子液体的热稳定性分别受杂原子-碳原子间作用 力和杂原子-氢键之间作用力所致，因此离子液体的分 解温度主要取决于其阴、阳离子结构及含水量. 从图 $3 \mathrm{a} \sim 3 \mathrm{~d}$ 中可以看出, 制备的 4 种 $\mathrm{SO}_{3} \mathrm{H}$-功能化离子液体 比较稳定. 离子液体在分解前, 热重曲线比较平坦, 在 $250{ }^{\circ} \mathrm{C}$ 以下几乎没有热失重，证明所制备的离子液体几 乎不挥发, 在失重过程中没有原料及其它杂质峰出现, 说明所制备的离子液体较纯，离子液体的热分解温度在 $250{ }^{\circ} \mathrm{C}$ 以上. 离子液体较好的热稳定性, 为很多反应提 供一个较大的操作温度范围. 与其它离子液体相比(表 2)，虽然咪唑、吡啶阳离子的热稳定性略高，但所合成 的 4 种已内酰胺离子液体对于酯化反应都有足够的热稳 定性，在酯化反应中均能稳定存在.

表 2 离子液体的热分解温度 ${ }^{a}$

Table 2 The thermal decomposition point of the ionic liquids

\begin{tabular}{cccccccc}
\hline IL & IL1 & IL2 & IL3 & IL4 & IL5 & IL6 & IL7 \\
\hline $\mathrm{DTG}_{\max } /{ }^{\circ} \mathrm{C}$ & 316 & 291 & 262 & 258 & 365 & 329 & 262 \\
\hline
\end{tabular}

${ }^{a}$ Test conditions: temperature range $20 \sim 500{ }^{\circ} \mathrm{C}$; scanning rate $10{ }^{\circ} \mathrm{C} / \mathrm{min}$.
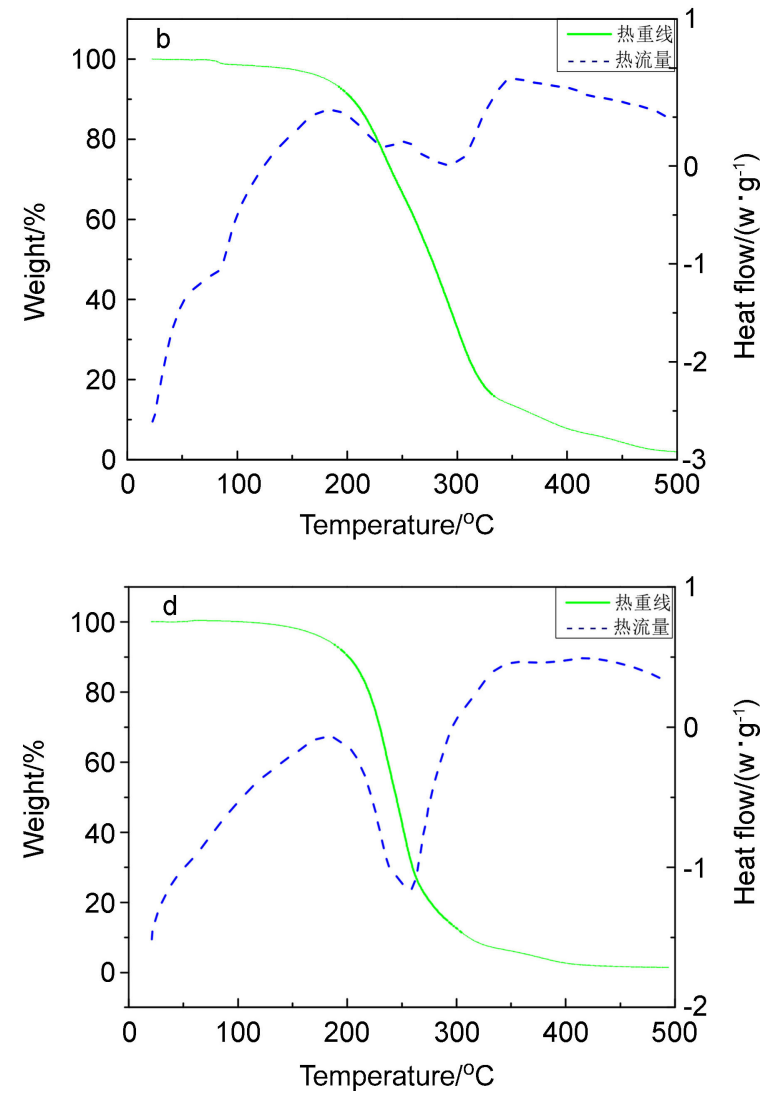

图 3 离子液体的 DSC-TG

Figure 3 DSC-TG of ionic liquid (a) $\left[\mathrm{C}_{3} \mathrm{SO}_{3} \mathrm{HCP}\right] \mathrm{HSO}_{4}$; (b) $\left[\mathrm{C}_{3} \mathrm{SO}_{3} \mathrm{HCP}\right] \mathrm{PTSA}$; (c) $\left[\mathrm{C}_{3} \mathrm{SO}_{3} \mathrm{HCP}\right] \mathrm{H}_{2} \mathrm{PO}_{4}$; (d) $\left[\mathrm{C}_{3} \mathrm{SO}_{3} \mathrm{HCP}_{\mathrm{BF}}\right.$ 


\section{4 离子液体溶解性分析}

离子液体溶解范围广, 能力强, 溶解度大, 且具备 较强的离子环境. 在许多有机和无机物质表现出良好的 溶解性, 而且具有介质和催化双重功能. 而离子液体的 溶解度取决于离子液体的本性. 表 3 是离子液体与其它 常用溶剂的相容性的测定结果. 结果表明, 离子液体与 非极性溶剂不混溶, 与较强的极性溶剂互溶, 如此类离 子液体均溶于水, 均不溶于有机羧酸酯. 与浓硫酸相比, 此类离子液体显著的优点是有利于反应的进行, 提高了 酯化反应的酯收率; 能够与酯产物自动分层, 简化了分 离过程; 与酯产物易分离, 可以循环使用. 因此, 离子 液体的溶解性能的研究为离子液体在催化酯化反应中 的应用提供了重要的数据支持.

\section{5 离子液体腐蚀性分析}

酸性离子液体对化学工业常用的反应容器、管道或 换热材料等的腐蚀性, 已成为其工业化应用无法回避的 问题, 近几年这方面的问题很受国内外学者的关 注 ${ }^{[18,19]}$. 腐蚀性作为离子液体的一个重要性质, 在很大 程度上决定了离子液体催化酯化反应过程的安全性和 经济性 ${ }^{[20]}$. 根据 $316 \mathrm{~L}$ 不锈钢在不同离子液体和浓硫酸 介质中的腐蚀速率测定结果(表 4)可知, 在相同条件下, 除了含 $\mathrm{BF}_{4}^{-}$阴离子的离子液体外, 离子液体的腐蚀性 顺序与与酸性顺序基本一致, 即离子液体酸性越强, 腐
蚀性越强, 但硫酸体系中的反应现象比离子液体的更为 剧烈. 尽管 $\left[\mathrm{C}_{3} \mathrm{SO}_{3} \mathrm{HCP}\right] \mathrm{BF}_{4}$ 比含其他阴离子的离子液体 低, 但其腐蚀性较高, 因为阴离子 $\mathrm{BF}_{4}^{-}$在溶液(含水)中 发生水解所致. 可见，离子液体的酸性尽管与硫酸接近, 但腐蚀性比硫酸要低，其腐蚀率不到硫酸的 $1 / 6$.

\section{6 离子液体的催化活性}

为了比较不同催化剂对酯化反应的催化活性, 本文 设计了一系列实验. 实验选择乙酸乙酯为反应体系, 反 应条件: 反应温度 $80{ }^{\circ} \mathrm{C}, n$ (乙醇) $: n$ (乙酸) $=1: 1.5$, 催 化剂用量为醇酸总质量的 $5 \%$, 反应时间 $6 \mathrm{~h}$ 的反应条件 下，考察不同酸性离子液体(ILs)对酯收率的影响，实验 结果如表 5 所示.

由表 5 可以看出, $\mathrm{SO}_{3} \mathrm{H}$-功能化离子液体对酯化反 应具有可与浓硫酸相比拟的催化活性, $\mathrm{HSO}_{4}^{-}$离子液体 的催化活性较高, 这与阴离子的酸性强弱相符合. 此外, 反应结束后离子液体均能与酯自动分层, 不仅简化了分 离过程，而且有利于平衡向酯化反应方向进行，这些都 是浓硫酸催化体系所不能比拟的. 虽然 4 种 $\mathrm{HSO}_{4}^{-}$离子 液体的酸性、腐蚀性和催化效果均相差不大，但合成己 内酰胺功能化离子液体的原料价格低廉, 不仅满足 “绿 色” 化学的要求, 而且降低了生产成本, 具有一定的经 济效益，适合工业化生产. 因此，我们选择合成廉价、环 境友好的离子液体 $\left[\mathrm{C}_{3} \mathrm{SO}_{3} \mathrm{HCP}\right] \mathrm{HSO}_{4}$ 作为酯化反应的催

表 3 不同离子液体的溶解性 ${ }^{a}$

Table 3 The solubility of different ionic liquids

\begin{tabular}{|c|c|c|c|c|c|}
\hline \multirow{2}{*}{ Ionic liquid } & \multicolumn{5}{|c|}{ Solubility of solvent } \\
\hline & Water & Methanol & Ethanol & Isopropanol & Acetone \\
\hline$\left[\mathrm{C}_{3} \mathrm{SO}_{3} \mathrm{HCP}\right] \mathrm{HSO}_{4}$ & $\mathrm{~s}$ & $\mathrm{~s}$ & $\mathrm{~s}$ & $\mathrm{~s}$ & $\mathrm{~s}$ \\
\hline$\left[\mathrm{C}_{3} \mathrm{SO}_{3} \mathrm{HCP}\right] \mathrm{PTSA}$ & $\mathrm{s}$ & $\mathrm{s}$ & $\mathrm{s}$ & $\mathrm{s}$ & $\mathrm{s}$ \\
\hline$\left[\mathrm{C}_{3} \mathrm{SO}_{3} \mathrm{HCP}\right] \mathrm{H}_{2} \mathrm{PO}_{4}$ & $\mathrm{~s}$ & $\mathrm{~s}$ & $\mathrm{~s}$ & $\mathrm{~s}$ & $\mathrm{~s}$ \\
\hline$\left[\mathrm{C}_{3} \mathrm{SO}_{3} \mathrm{HCP}\right] \mathrm{BF}_{4}$ & $\mathrm{~s}$ & $\mathrm{~s}$ & $\mathrm{~s}$ & $\mathrm{~s}$ & $\mathrm{~s}$ \\
\hline $\mathrm{H}_{2} \mathrm{SO}_{4}$ & $\mathrm{~s}$ & $\mathrm{~s}$ & $\mathrm{~S}$ & $\mathrm{~s}$ & $\mathrm{~s}$ \\
\hline \multirow{2}{*}{ Ionic liquid } & \multicolumn{5}{|c|}{ Solubility of solvent } \\
\hline & Ether & Ethyl acetate & Cyclohexane & Methylbenzene & Trichloromethane \\
\hline$\left[\mathrm{C}_{3} \mathrm{SO}_{3} \mathrm{HCP}\right] \mathrm{HSO}_{4}$ & $\mathrm{i}$ & $\mathrm{i}$ & $\mathrm{i}$ & $\mathrm{i}$ & $\mathrm{i}$ \\
\hline$\left[\mathrm{C}_{3} \mathrm{SO}_{3} \mathrm{HCP}\right] \mathrm{PTSA}$ & $\mathrm{i}$ & $\mathrm{i}$ & $\mathrm{i}$ & $\mathrm{i}$ & $\mathrm{i}$ \\
\hline$\left[\mathrm{C}_{3} \mathrm{SO}_{3} \mathrm{HCP}\right] \mathrm{H}_{2} \mathrm{PO}_{4}$ & $\mathrm{i}$ & $\mathrm{i}$ & $\mathrm{i}$ & $\mathrm{i}$ & $\mathrm{i}$ \\
\hline$\left[\mathrm{C}_{3} \mathrm{SO}_{3} \mathrm{HCP}\right] \mathrm{BF}_{4}$ & $\mathrm{i}$ & $\mathrm{i}$ & $\mathrm{i}$ & $\mathrm{i}$ & $\mathrm{i}$ \\
\hline $\mathrm{H}_{2} \mathrm{SO}_{4}$ & $\mathrm{~s}$ & $\mathrm{~s}$ & $\mathrm{~s}$ & $\mathrm{~s}$ & $\mathrm{~s}$ \\
\hline
\end{tabular}

${ }^{a}$ Test conditions: $V($ solvent $)=5 \mathrm{~mL}, m\left(\mathrm{IL}\right.$ or $\left.\mathrm{H}_{2} \mathrm{SO}_{4}\right)=0.2$ g, r.t.; s: solubility; i: insolubility.

表 4 离子液体的腐蚀性 ${ }^{a}$

Table 4 Corrosion of ILs for Austenitic stainless steel 316 after immersion

\begin{tabular}{|c|c|c|c|c|c|c|}
\hline Entry & IL & $m(\mathrm{IL}) / \mathrm{g}$ & $t / \mathrm{h}$ & $T /{ }^{\circ} \mathrm{C}$ & Mass loss/g & Corrosion rate $/\left(\mathrm{g} \cdot \mathrm{m}^{-2} \cdot \mathrm{h}^{-1}\right)$ \\
\hline 1 & {$\left[\mathrm{C}_{3} \mathrm{SO}_{3} \mathrm{HCP}\right] \mathrm{HSO}_{4}$} & 1.36 & 6 & 80 & 0.0183 & 3.63 \\
\hline 2 & {$\left[\mathrm{C}_{3} \mathrm{SO}_{3} \mathrm{HCP}\right] \mathrm{PTSA}$} & 1.36 & 6 & 80 & 0.0178 & 3.53 \\
\hline 3 & {$\left[\mathrm{C}_{3} \mathrm{SO}_{3} \mathrm{HCP}\right] \mathrm{H}_{2} \mathrm{PO}_{4}$} & 1.36 & 6 & 80 & 0.0160 & 3.17 \\
\hline 4 & {$\left[\mathrm{C}_{3} \mathrm{SO}_{3} \mathrm{HCP}\right] \mathrm{BF}_{4}$} & 136 & 6 & 80 & 0.0258 & 5.12 \\
\hline 5 & $\mathrm{H}_{2} \mathrm{SO}_{4}$ & 136 & 6 & 80 & 0.0942 & 18.69 \\
\hline
\end{tabular}

${ }^{a}$ Test conditions: $n\left(\mathrm{C}_{2} \mathrm{H}_{5} \mathrm{OH}\right)=0.2 \mathrm{~mol}, n\left(\mathrm{CH}_{3} \mathrm{COOH}\right)=0.3 \mathrm{~mol}, w(\mathrm{IL})=5 \%$. 
表 5 离子液体的催化活性 ${ }^{a}$

Table 5 Effect of different catalysts on results of esterification

\begin{tabular}{clc||ccc}
\hline Entry & \multicolumn{1}{c||}{$\mathrm{IL}$} & Yield/\% & Entry & \multicolumn{1}{c}{ IL } & \multicolumn{2}{c}{ Yield/\% } \\
\hline 1 & {$\left[\mathrm{C}_{3} \mathrm{SO}_{3} \mathrm{Hmim}\right] \mathrm{HSO}_{4}$} & 80.2 & 5 & {$\left[\mathrm{C}_{3} \mathrm{SO}_{3} \mathrm{HCP}\right] \mathrm{PTSA}$} & 72.1 \\
2 & {$\left[\mathrm{C}_{3} \mathrm{SO}_{3} \mathrm{HPy}\right] \mathrm{HSO}_{4}$} & 70.4 & 6 & {$\left[\mathrm{C}_{3} \mathrm{SO}_{3} \mathrm{HCP}_{\mathrm{H}} \mathrm{PO}_{4}\right.$} & 60.7 \\
3 & {$\left[\mathrm{C}_{3} \mathrm{SO}_{3} \mathrm{Hnhm}\right] \mathrm{HSO}_{4}$} & 83.2 & 7 & {$\left[\mathrm{C}_{3} \mathrm{SO}_{3} \mathrm{HCP}_{\mathrm{BF}}\right.$} & 73.6 \\
4 & {$\left[\mathrm{C}_{3} \mathrm{SO}_{3} \mathrm{HCP}\right] \mathrm{HSO}_{4}$} & 79.9 & 8 & $\mathrm{H}_{2} \mathrm{SO}_{4}$ & 80.1 \\
\hline
\end{tabular}

${ }^{a}$ Reaction conditions: $n\left(\mathrm{C}_{2} \mathrm{H}_{5} \mathrm{OH}\right): n\left(\mathrm{CH}_{3} \mathrm{COOH}\right)=1: 1.5, w\left(\mathrm{IL}\right.$ or $\left.\mathrm{H}_{2} \mathrm{SO}_{4}\right)=5 \%, T=80{ }^{\circ} \mathrm{C}, t=6 \mathrm{~h}$.

化剂进行下一步研究.

\section{7 正交实验考察 $\left[\mathrm{C}_{3} \mathrm{SO}_{3} \mathrm{HCP}\right] \mathrm{HSO}_{4}$ 对酯收率的影响}

本实验选用三水平四因素的正交表，四因素分别为 反应温度、催化剂用量、醇酸摩尔比、反应时间，每个 因素选择 3 个水平. 评价指标为乙酸乙酯产品收率, 选 用催化剂为 $\left[\mathrm{C}_{3} \mathrm{SO}_{3} \mathrm{HCP}\right] \mathrm{HSO}_{4}$, 所设计的正交实验因素水平表(表 6)以及实验结果表(表 7).

表 6 正交实验因素-水平表

Table 6 Levels and factors for orthogonal experiment

\begin{tabular}{ccccc}
\hline Level & Temp. $/{ }^{\circ} \mathrm{C}$ & $\begin{array}{c}\text { Amount of } \\
\text { catalyst } /(m \bullet m)\end{array}$ & Mole ratio & $\begin{array}{c}\text { Reaction } \\
\text { time } / \mathrm{h}\end{array}$ \\
\hline 1 & 70 & $3 \%$ & $1: 1.0$ & 4 \\
2 & 75 & $5 \%$ & $1: 1.5$ & 5 \\
3 & 80 & $8 \%$ & $1: 2.0$ & 6 \\
\hline
\end{tabular}

由表 7 可以看出, 在离子液体 $\left[\mathrm{C}_{3} \mathrm{SO}_{3} \mathrm{HCP}\right] \mathrm{HSO}_{4}$ 催 化合成乙酸乙酯的实验研究中, 四种因素对乙酸乙酯收 率均有影响, 反应温度是影响收率的最大因素, 其次为 醇酸物质的量比和催化剂用量, 反应时间对产品收率影 响最小. 此结果表明, 温度是影响反应的重要因素, 因 为酯化反应是可逆反应，所以热力学因素是影响反应的 主要因素. 而催化剂只影响动力学因素, 只要催化剂没 有大的损失，酯的是收率是不会受大的影响的，因此动 力学因素相对不重要. 根据实验结果直观分析可知, 离
子液体 $\left[\mathrm{C}_{3} \mathrm{SO}_{3} \mathrm{HCP}\right] \mathrm{HSO}_{4}$ 催化合成乙酸乙酯的最佳工艺 条件为: 醇酸物质的量比 $1: 1.5$, 催化剂用量为酸醇总 质量的 $5 \%$, 反应时间 $6 \mathrm{~h}$, 反应温度为 $80{ }^{\circ} \mathrm{C}$. 在此工艺 条件下，乙酸乙酯的收率可达 $93.8 \%$.

\section{8 离子液体的重复使用性能}

离子液体较传统催化剂的显著优点即可循环使用, 反应结束后离子液体与产品酯自动分层, 将分离出来的 离子液体减压蒸馏充分干燥后回收使用. 在反应温度 $80{ }^{\circ} \mathrm{C}, n$ (乙醇) $: n$ (乙酸) $=1: 1.5$, 催化剂 $\left(\left[\mathrm{C}_{3} \mathrm{SO}_{3} \mathrm{HCP}\right]-\right.$ $\mathrm{HSO}_{4}$ ) 用量为醇酸总质量的 $5 \%$, 反应时间 $6 \mathrm{~h}$ 的最佳反 应条件下，考察离子液体的重复使用性能，结果如图 4.

从图 4 中可以看出, 随着离子液体重复使用次数的 增加，乙酸乙酯的收率依然很高，即重复使用 10 次后， 活性仅略有下降, 催化活性的降低是由于在对离子液体 回收处理的过程中略有损失导致产率略有下降，说明离 子液体具有较好的重复使用性能. 对重复使用 10 次的 离子液体干燥处理后进行表征, 发现重复使用 10 次的 离子液体的红外谱图中主要特征峰与最初的离子液体 的红外谱图中的特征峰基本吻合，没有新的特征峰出 现，而且经重复使用 10 次的离子液体的热分解温度基 本维持在 $250{ }^{\circ} \mathrm{C}$. 可见, 干燥后的离子液体可以保持良 好的重复使用性能.

表 7 正交实验与结果

Table 7 Results of orthogonal experiment

\begin{tabular}{|c|c|c|c|c|c|}
\hline Run & $\begin{array}{c}\text { A } \\
\text { Temperature } /{ }^{\circ} \mathrm{C}\end{array}$ & $\begin{array}{c}\text { B } \\
\text { Amount of catalyst } /(m / m)\end{array}$ & $\begin{array}{c}\mathrm{C} \\
\text { Molar ratio }\end{array}$ & $\begin{array}{c}\mathrm{D} \\
\text { Reaction time/h }\end{array}$ & Yield/\% \\
\hline 1 & 70 & $3 \%$ & $1: 1.0$ & 4 & 53.6 \\
\hline 2 & 70 & $5 \%$ & $1: 1.5$ & 5 & 82.4 \\
\hline 3 & 70 & $8 \%$ & $1: 2.0$ & 6 & 75.1 \\
\hline 4 & 75 & $3 \%$ & $1: 1.5$ & 6 & 85.9 \\
\hline 5 & 75 & $5 \%$ & $1: 2.0$ & 4 & 88.6 \\
\hline 6 & 75 & $8 \%$ & $1: 1.0$ & 5 & 73.8 \\
\hline 7 & 80 & $3 \%$ & $1: 2.0$ & 5 & 80.7 \\
\hline 8 & 80 & $5 \%$ & $1: 1.0$ & 6 & 86.5 \\
\hline 9 & 80 & $8 \%$ & $1: 1.5$ & 4 & 90.3 \\
\hline K1 & 70.367 & 73.400 & 71.300 & 77.500 & - \\
\hline $\mathrm{K} 2$ & 82.767 & 85.833 & 86.200 & 78.967 & - \\
\hline $\mathrm{K} 3$ & 85.833 & 79.733 & 81.467 & 82.500 & - \\
\hline $\mathrm{R}$ & 15.466 & 12.433 & 14.900 & 5.000 & - \\
\hline \multicolumn{3}{|c|}{ Optimal conditions } & $\begin{array}{ll}\mathrm{C}_{2} & \mathrm{D} \\
\end{array}$ & & \\
\hline
\end{tabular}




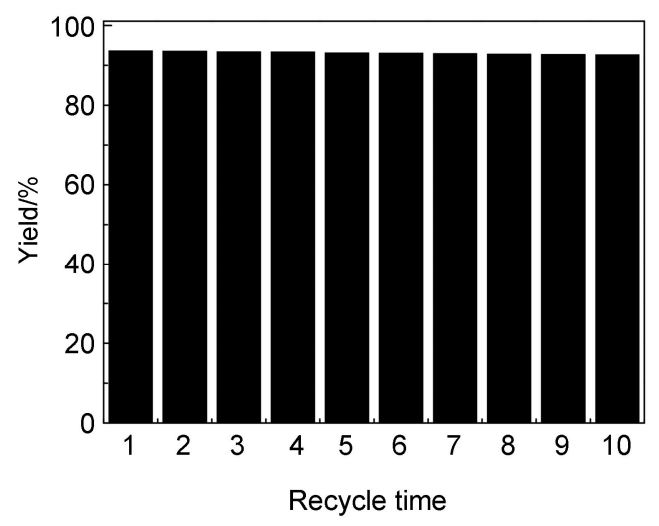

图 4 离子液体的重复使用性能

Figure 4 Reusability results of ionic liquids

\section{9 离子液体催化合成系列乙酯}

以离子液体 $\left[\mathrm{C}_{3} \mathrm{SO}_{3} \mathrm{HCP}\right] \mathrm{HSO}_{4}$ 为催化剂, 对催化合 成系列乙酯进行了研究, 结果如表 8. 由表 8 可以看出, 该离子液体对催化正丁酸、正己酸、月桂酸、草酸、丁 二酸、马来酸与乙醇的酯化反应均具有较高的催化效果, 其系列乙酯的收率均在 $80 \%$ 以上, 但是如果对酯化反应 条件做进一步的优化有望提高酯收率, 达到更好的催化 效果. 反应结束后, 离子液体与系列乙酯均能自动分层, 后处理简单，可以循环使用。

表 8 离子液体催化合成系列乙酯 ${ }^{a}$

Table 8 Esterification results for various acids with ethanol in ionic liquid

\begin{tabular}{clccc}
\hline Entry & \multicolumn{1}{c}{ Acid } & $n$ (acid) $: n$ (ethanol) $)$ & $T /{ }^{\circ} \mathrm{C}$ & Yield $/ \%$ \\
\hline 1 & $n$-Butyric acid & $1: 2.0$ & 80 & 89.4 \\
2 & $n$-Hexanoic acid & $1: 2.0$ & 85 & 90.8 \\
3 & Lauric acid & $1: 2.0$ & 85 & 88.7 \\
4 & Oxalic acid & $1: 2.5$ & 90 & 85.6 \\
5 & Succinic acid & $1: 3.0$ & 100 & 83.9 \\
6 & Maleic & $1: 3.0$ & 100 & 91.2 \\
\hline
\end{tabular}

${ }^{a}$ Reaction condition: $n($ acid $)=0.1 \mathrm{~mol} ; w(\mathrm{IL})=5 \%, t=6 \mathrm{~h}$.

\section{2 结论}

根据离子液体的阴离子的不同, 采用两步法合成了 4 种不同催化性能的离子液体, 并对乙酸乙酯的合成进 行了应用研究. 与传统浓硫酸催化酯化相比, 该催化体 系的优势在于: (1)此类离子液体的酸性与其催化活性有 关, 酸性越强, 其催化活性越高, 少量的离子液体就可 以达到高酯收率; (2)该类离子液体催化工艺具有成本 低, 过程清洁, 而且不含 $\mathrm{F} 、 \mathrm{Cl} 、 \mathrm{Br}$ 等卤素原子, 没有对 环境潜在的污染; (3)产物酯与离子液体可以自动分层, 经简单的倾析法分离出来, 可以循环使用, 而且仍具有 较高的催化活性, 显示了离子液体作为酯化反应催化剂 所具有的巨大优势; (4)该体系反应条件温和奥氏体型 $316 \mathrm{~L}$ 不锈钢的浸渍试验结果表明, 所研究的酸性离子
液体腐蚀性比浓硫酸低, 有利于延长反应装置材料的使 用寿命，提高了反应设备的运行周期，说明离子液体具 有替代传统浓硫酸应用于工业酯化反应的潜在价值. 综 上所述，离子液体既可以作溶剂又可以作催化剂，解决 了传统酸催化剂所具有的副产物多、易挥发、腐蚀性强 和用量大等缺陷, 有望替代传统酸催化剂应用于酯化反 应过程，为真正实现绿色化学工业化提供了可靠的基 础, 具有璀骤的应用前景.

\section{3 实验部分}

\section{1 仪器与试剂}

RE-2000B 型旋转蒸发器(上海亚荣生化仪器厂); DF-101S 型集热式恒温加热磁力搅拌器(长城科工贸有 限公司); FTS-135 型傅立叶变换红外光谱仪 (美国 BIO-RAD 公司); Bruker Avance 500MHz 型核磁共振仪 (瑞士 BRUKER 公司); STD-2960 型热重分析仪(德国 Netzsch 公司); PHS-25 型 $\mathrm{pH}$ 计(上海精密科学仪器有 限公司).

己内酰胺 (分析纯, 天津市光复精细化工研究所); 1,3-丙烷磺酸内酯(分析纯，百顺(北京)化学科技有限公 司); 浓硫酸(分析纯，大茂化学(天津)制剂有限公司); 对甲苯磺酸(分析纯, 天津博迪化工股份有限公司); 磷 酸(分析纯, 天津市东方化工); 嗍酸(分析纯, 石家庄炼 油厂生产); 乙酸(分析纯, 永大化学制剂(天津)有限公 司); 乙醇(分析纯, 天津市红岩试剂厂).

\section{2 己内酰胺功能化离子液体的制备}

\subsubsection{1-(3-磺丙基)已内酰胺盐的制备}

在配有机械搅拌的 $250 \mathrm{~mL}$ 三口烧瓶中加入 $13.3 \mathrm{~g}$ (0.1 mol) 己内酰胺和 $10 \mathrm{~mL}$ 水, 待充分溶解, 再加入 $12.2 \mathrm{~g}(0.1 \mathrm{~mol}) 1,3$-丙烷磺酸内酯, 室温下反应 $24 \mathrm{~h}$ 以 上，得离子液体中间体 1-(3-磺丙基)己内酰胺盐，产率 大于 $98 \%$, 参照文献 $[21]$.

\subsection{2 已内酰胺类 $\mathrm{SO}_{3} \mathrm{H}$-功能化离子液体的制备}

将 1-(3-磺丙基)己内酰胺盐分别与硫酸、对甲苯磺 酸、磷酸、氟硼酸按等摩尔混合, 加入一定量溶剂乙腈, 回流反应 $6 \mathrm{~h}$ 以上, 然后对产物进行提纯处理, 得到离 子液体 IL1 IL4.

合成的己内酰胺类离子液体核磁谱图数据(参考文 献[22])如下:

$\left[\mathrm{C}_{3} \mathrm{SO}_{3} \mathrm{HCP}\right] \mathrm{HSO}_{4}:{ }^{1} \mathrm{H}$ NMR $\left(500 \mathrm{MHz}, \mathrm{D}_{2} \mathrm{O}\right) \delta$ : $1.596 \sim 1.617(\mathrm{~m}, 6 \mathrm{H}), 1.740 \sim 1.752(\mathrm{~m}, 2 \mathrm{H}), 2.475(\mathrm{t}$, $J=11.5 \mathrm{~Hz}, 2 \mathrm{H}), 2.933 \sim 3.015$ (m, 2H), 3.244 (t, $J=10.5$ $\mathrm{Hz}, 2 \mathrm{H}), 3.665 \sim 3.691(\mathrm{~m}, 2 \mathrm{H})$.

$\left[\mathrm{C}_{3} \mathrm{SO}_{3} \mathrm{HCP}\right]$ PTSA: ${ }^{1} \mathrm{HNMR}\left(500 \mathrm{MHz}, \mathrm{D}_{2} \mathrm{O}\right) \delta$ : 
$1621 \sim 1.648(\mathrm{~m}, 6 \mathrm{H}), 1.766 \sim 1.777(\mathrm{~m}, 2 \mathrm{H}), 2.411(\mathrm{~s}$, $2 \mathrm{H}), 2.487(\mathrm{t}, J=11.5 \mathrm{~Hz}, 3 \mathrm{H}), 2.965 \sim 2.996(\mathrm{~m}, 2 \mathrm{H})$, $3.260(\mathrm{t}, J=10.5 \mathrm{~Hz}, 2 \mathrm{H}), 3.696 \sim 3.722(\mathrm{~m}, 2 \mathrm{H}), 7.386(\mathrm{~d}$, $J=8 \mathrm{~Hz}, 2 \mathrm{H}), 7.705(\mathrm{~d}, J=8.5 \mathrm{~Hz}, 2 \mathrm{H})$.

$\left[\mathrm{C}_{3} \mathrm{SO}_{3} \mathrm{HCP}\right] \mathrm{H}_{2} \mathrm{PO}_{4}:{ }^{1} \mathrm{HNMR}\left(500 \mathrm{MHz}, \mathrm{D}_{2} \mathrm{O}\right) \delta$ : $1.597 \sim 1.647(\mathrm{~m}, 6 \mathrm{H}), 1.753 \sim 1.764(\mathrm{~m}, 2 \mathrm{H}), 2.477(\mathrm{t}$, $J=16.5,2 \mathrm{H}), 2.950 \sim 2.981(\mathrm{~m}, 2 \mathrm{H}), 3.250(\mathrm{t}, J=10.5 \mathrm{~Hz}$, $2 \mathrm{H}), 3.619 \sim 3.707(\mathrm{~m}, 2 \mathrm{H})$.

$\left[\mathrm{C}_{3} \mathrm{SO}_{3} \mathrm{HCP}\right] \mathrm{BF}_{4}:{ }^{1} \mathrm{HNMR}\left(500 \mathrm{MHz}, \mathrm{D}_{2} \mathrm{O}\right) \delta: 1.601$ (m, 6H), $1.745 \sim 1.756(\mathrm{~m}, 2 \mathrm{H}), 2.478(\mathrm{t}, J=11.5 \mathrm{~Hz}, 2 \mathrm{H})$, $2.938 \sim 2.969(\mathrm{~m}, 2 \mathrm{H}), 3.247(\mathrm{t}, J=10 \mathrm{~Hz}, 2 \mathrm{H}), 3.607 \sim$ $3.695(\mathrm{~m}, 2 \mathrm{H})$.

1-甲基-3-(3-磺丙基)咪唑硫酸氢盐 $\left(\left[\mathrm{C}_{3} \mathrm{SO}_{3} \mathrm{Hmim}\right]-\right.$ $\mathrm{HSO}_{4}$ )、1-(3-磺丙基)吡啶硫酸氢盐( $\left[\mathrm{C}_{3} \mathrm{SO}_{3} \mathrm{Hpy}\right] \mathrm{HSO}_{4}$ ) 和 4-(3-磺丙基)吗啡啉硫酸氢盐 $\left(\left[\mathrm{C}_{3} \mathrm{SO}_{3} \mathrm{Hnhm}\right] \mathrm{HSO}_{4}\right)$ 分别 参照文献 $[23,24]$ 的方法合成.

\section{3 离子液体的表征和性能测定}

(1)酸性测定. 在室温条件下, 对质量浓度分别为 $1.0 \%, 2.0 \%, 3.0 \%, 4.0 \%, 5.0 \%$ 的 4 种已内酰胺功能化离 子液体和浓硫酸水溶液 $\mathrm{pH}$ 值进行了测试.

(2)结构表征. 采用 FTS-135 型傅立叶变换红外光谱 仪对离子液体结构的官能团进行测定; 采用 Bruker Avance $500 \mathrm{MHz}$ 核磁共振波谱仪, 采用 $\mathrm{D}_{2} \mathrm{O}$ 为溶剂检测 氢原子的化学位移.

(3)稳定性测试. 用 STD-2960 差热-热重联用热分 析仪测试离子液体的热稳定性, 热重分析条件: 气体是 $\mathrm{N}_{2}$, 升温速率: $10{ }^{\circ} \mathrm{C} / \mathrm{min}$.

(4)溶解性测定. 取少量所合成的离子液体分别溶 于 $5 \mathrm{~mL}$ 的不同溶剂中, 充分摚拌后观察其溶解程度. 所用的溶剂为: 水、甲醇、乙醇、异丙醇、丙酮、甲苯、 乙醚、氯仿、乙酸乙酯、环己烷.

(5)腐蚀性考察. 实验采用奥氏体型 $316 \mathrm{~L}$ 不锈钢板 (上海宝钢集团, $20 \mathrm{~mm} \times 20 \mathrm{~mm} \times 0.5 \mathrm{~mm}$ ) 作为试验钢 样, 考察其在 4 种已内酰胺功能化离子液体为催化剂的 酯化体系中离子液体的腐蚀性, 并与浓 $\mathrm{H}_{2} \mathrm{SO}_{4}$ 的催化体 系进行对比. 实验前用砂纸对钢片逐级打磨, 然后分别 用乙醇、丙酮、二次蒸馏水清洗. 实验条件为: 在 $250 \mathrm{~mL}$ 的三口烧瓶中分别加入 $0.3 \mathrm{~mol}$ 乙酸、 $0.15 \mathrm{~mol}$ 乙醇和 一定量离子液体或浓硫酸, $80{ }^{\circ} \mathrm{C}$ 条件下磁力搅拌 $6 \mathrm{~h}$, 试样浸渍前后的处理参照国际 GB/T4334.6-2000《不锈 钢 5\%硫酸腐蚀试验方法》进行, 得到试验钢板反应前 后的质量差, 再根据试验钢板的表面积, 最后计算得到 $316 \mathrm{~L}$ 不锈钢板的腐蚀速率.

\section{4 酯化反应}

准确称量 $0.1 \mathrm{~mol}(4.6 \mathrm{~g})$ 乙醇、 $0.15 \mathrm{~mol}(9.0 \mathrm{~g})$ 乙酸 和 $0.68 \mathrm{~g}$ (酸醇总质量的 $5 \%$ ) 已内酰胺功能化离子液体 装入带有回流冷凝管的四口烧瓶中, 在所设温度 $80{ }^{\circ} \mathrm{C}$ 下磁力搅拌 $6 \mathrm{~h}$ 后, 冷却至室温, 反应后的混合溶液分 层, 上层为乙酸乙酯混合溶液, 蒸馏收集产品馏分; 下 层为离子液体, 经简单的减压旋蒸回收重复使用.

辅助材料(Supporting Information) 己内酰胺功能化 离子液体的 ${ }^{1} \mathrm{H}$ NMR. 这些材料可以免费从本刊网站 (http://sioc-journal.cn/)上下载.

\section{References}

[1] Li, X.; Lin, Q.; Ma, L. Ultrason. Sonochem. 2010, 17, 752.

[2] Zhao, D. S.; Liu, M. S.; Xu, Z. C.; F, J. T.; Ren, P. B. Chem. Ind. Eng. Prog. 2011, 30, 2287 (in Chinese).

(赵地顺, 刘猛帅, 徐智策, 付江涛, 任培兵, 化工进展, 2011, 30, 2287.)

[3] Zhao, D. B.; Fei, Z. F.; Geldbach, T. J.; Scopelliti, R.; Laurenczy, G.; Dyson, p. J. J. Am. Chem. Soc. 2005, 88, 665.

[4] Inui, K.; Kurabayashi, T.; Sato, S.; Ichikawa, N. J. Mol. Catal. A: Chem. 2004, 216, 147.

[5] Zhao, D. S.; Liu, M. S.; Ge, J. J.; Zhang, J.; Ren, P. B. Chin. J. Org. Chem. 2012, 32, 2382 (in Chinese).

(赵地顺, 刘猛帅, 葛京京, 张娟, 任培兵, 有机化学, 2012, 32, 2382.)

[6] Li, Y.; Hu, S. L.; Cheng, J. H.; Lou, W. Y. Chin. J. Catal. 2014, 35, 396.

[7] Song, Y. L.; Wang, X. C.; Huang, C. P.; Liang, F. B.; Liu, Z. C.; Chen, B. H. Chin. J. Org. Chem. 2013, 33, 1715 (in Chinese). (宋彦磊, 王新承, 黄崇品, 梁凤兵, 毓志超, 陈标华, 有机化学, 2013, 33, 1715.)

[8] Zhao, D. W.; Wu, Y. T.; Chen, T. T.; Dai, L. Y.; Wang, Y. Y. Chin. J. Org. Chem. 2013, 33, 1791 (in Chinese).

(赵东旺, 吴悦粀, 陈婷婷, 戴立益, 王媛媛, 有机化学, 2013, 33, 1791.)

[9] Gui, J. Z.; Ban, H. Y.; Cong, X. H.; Zhang, X. T.; Hu, Z. D.; Sun, Z. L. J. Mol. Catal. A: Chem. 2005, 225, 27.

[10] Cai, X. J.; Cui, S. H.; Qu, L. P.; Yuan, D. D.; Lu, B.; Cai, Q. H. Catal. Commun. 2008, 9, 1173.

[11] Shi, F.; Zhang, Q. H.; Li, D. M.; Deng, Y. Q. Chem.-Eur. J. 2008, 11,5279 .

[12] Dawood, E.; Babak, K.; Abbas, M.; Javad, R. ChemPlusChem 2014, 79, 1147.

[13] James, H. C.; Thomas, J. F.; Duncan, J. M.; James, S. Sustainable Chem. Processes 2013, 1(13), 1.

[14] Han, X. X.; Du, H.; Hung, C. T.; Liu, L. L.; Wu, P. H.; Ren, D. H.; Huang, S. J.; Liu, S. B. Green Chem. 2015, 17(1), 499.

[15] Li, Z.; Zhao, Y. W.; Han, F.; Yang, L.; Song, H. Y.; Chen, J.; Xia, C. G. Sci. China: Chem. 2012, 42(4), 502 (in Chinese). (李臻, 赵应伟, 韩峰, 杨否, 宋河远, 陈静, 夏春谷, 中国科学: 化学, 2012, 42(4), 502.)

[16] Swatloski, R. P.; Holbrey, J. D.; Memon, S. B.; Caldwell, G. A.; Caldwell, K. A. Rogers, R. D. Chem. Commun. 2004, 668.

[17] Du, Z.; Li, Z.; Zhang, J.; Zhu, L.; Deng, Y. J. Phys. Chem. B 2005, 109, 19542.

[18] Duran, A.; Jimenez De Haro, M. C.; Perezrodriguez, J. L.; Franqe- 
lo, M. L.; Herrera, L. K.; Justo, A. Appl. Surf. Sci. 2007, 253(17), 7295.

[19] Ilaria, P.; Ugo, B.; Stefano, C.; Alessio, F.; Alessandro, L. Sol. Energy Mater. Sol. Cells 2008, 92, 510.

[20] Lv, X. M. M. S. Thesis, Nanjing University, Nanjing, 2011 (in Chinese)

(吕学铭, 硕士论文, 南京大学, 南京, 2011.)

[21] Tan, W. Y.; Zhang, Z. H.; Chen, Q.; He, M. Y. Chem. Ind. Eng. Prog. 2012, 31, 896 (in Chinese).

(谭维一，张致慧，陈群，何明阳，化工进展，2012，31,896.)
[22] Gao, H. B. Organic Chemistry, Higher Education Press, Beijing, 2005, p. 301 (in Chinese).

(高鸿宾，有机化学，高等教育出版社，北京， 2005, p. 301.)

[23] Ge, J. J. M. S. Thesis, Hebei University of Science and Technology, Hebei, 2014 (in Chinese).

(葛京京, 硕士论文, 河北科技大学, 河北, 2014.)

[24] Huang, B. H.; Li, Z. J.; Shi, N.; Xu, X. L.; Fang, Y. X. Chin. J. Org. Chem. 2009, 29, 770 (in Chinese).

(黄宝华, 黎子进, 史娜, 徐效陵, 张焜, 方岩雄, 有机化学, 2009, 29, 770.)

(Li, L.; Fan, Y.) 